\title{
A trajetória da política nacional de reorientação da formação profissional em saúde no SUS
}

\author{
The trajectory of the national policy for the reorientation \\ of professional training in health in the Unified Health System (SUS)
}

${ }^{1}$ Subsecretaria de Atenção à Saúde, Secretaria de Estado de Saúde do Rio de Janeiro. R. México 128/1.103 Centro. 20031-142 Rio de Janeiro RJ.

hendias@hotmail.com ${ }^{2}$ Departamento de Administração e Planejamento em Saúde, Escola Nacional de Saúde Pública, Fundação Oswaldo Cruz.

\begin{abstract}
This paper examines the national pol$i c y$ and its antecedents for reorientation of professional health training implemented after 2003. It highlights landmarks and transformations in the course of policies between 1980 and 2010, elements of continuity and change and the connections between past and current policy initiatives. The study involved a review of the literature on the subject and document analysis supported by theoretical analysis of public policies, particularly historical institutionalism. The results point to four different moments during the trajectory of the policy, marked by changes in the initiatives of reorientation of higher education in health: antecedents; initial experiences; university protagonism; broadening and enhancement. As an element of continuity, there is the permanence of objects in the guiding principles advocated in the policies. The evidence of implementation expresses prospects of enhancement, with diversification of mobilized actors and organizations, and more projects implemented. The accumulated experience suggests structural maturity of the structural bases of action and the main changes relate to the enhancement of decision-making bodies of the SUS and the approximation to the process of decentralization and regionalization of national health policy.
\end{abstract}

Key words Training in human resources, $\mathrm{Hi}$ gher education, Public health policies
Resumo Este artigo analisa a política nacional de reorientação da formação profissional em saúde, desenvolvida a partir de 2003, e seus antecedentes. Destaca marcos e transformações no percurso temporal da política de 1980 a 2010, elementos de continuidade e mudanças e conexões existentes entre iniciativas anteriores e a politica atual. O estudo envolveu revisão de literatura sobre o tema e análise documental apoiados no referencial teórico de análise das políticas públicas, em especial o institucionalismo histórico. Os resultados apontam quatro distintos momentos da trajetória da politica, demarcados por inflexões nas iniciativas de reorientação da formação superior em saúde: antecedentes; experiências iniciais; protagonismo universitário; ampliação e aprimoramento. Como elemento de continuidade, nota-se permanência dos eixos norteadores nos objetos preconizados nas políticas. As evidências de implementação expressam perspectiva de ampliação, com diversificação dos atores e organizações mobilizados, e mais projetos implantados. A experiência acumulada sugere amadurecimento das bases estruturantes das ações e as principais mudanças referem-se à valorização das instâncias decisórias do SUS e à aproximação com o processo de descentralização e regionalização da política nacional de saúde.

Palavras-chave Formação de recursos humanos, Ensino superior, Políticas públicas de saúde 


\section{Introdução}

As preocupações em torno da gestão do trabalho e da educação em saúde se fazem presentes nas análises das políticas de saúde no Brasil. Os estudos sugerem a presença inconstante do tema nos espaços de construção das políticas, embora as limitações do ensino e as inadequações do perfil profissional frente às necessidades de saúde da população já lograrem-se evidentes desde a segunda metade dos anos $70^{1-4}$. Ainda assim, há mais de duas décadas as questões relacionadas à formação profissional constituem-se objeto de discussão das conferências nacionais de saúde e de recursos humanos e compõem os textos referentes à legislação do Sistema Único de Saúde (SUS) e suas bases normativas ${ }^{5}$.

Trabalhos recentes enfatizam que as políticas de gestão da educação em saúde tomaram novo fôlego a partir de 2003 com a criação da Secretaria de Gestão do Trabalho e da Educação na Saúde (SGTES) no Ministério da Saúde, resultando na aproximação estratégica entre saúde e educação ${ }^{1,6,7}$. Foram deflagradas iniciativas visando ampliar a qualificação da força de trabalho por meio de ações de educação permanente que entre outros objetivos, articulam a formação profissional às práticas dos serviços de saúde ${ }^{7}$.

No que se refere à formação profissional em saúde, as ações da SGTES incluem, dentre outras, a indução de mudanças nas graduações, articuladas pelas universidades e os serviços de saúde, reunidas na política nacional de reorientação da formação profissional em saúde. Os principais eixos da política são a integração ensino-serviço com ênfase na atenção básica; a integralidade em saúde como eixo reorientador das práticas no processo de formação e qualificação dos profissionais para o SUS; e a reformulação do projeto político-pedagógico dos cursos de graduação baseada nas Diretrizes Curriculares Nacionais ${ }^{8,9}$.

Este artigo analisa a política nacional de reorientação da formação profissional em saúde, tendo como marco a criação da Secretaria de Gestão do Trabalho e da Educação na Saúde. Considera-se que o processo de formulação de políticas no setor tem sido condicionado pelo caráter tardio da aproximação entre o Ministério da Saúde e da Educação e pelos desafios relacionados ao ordenamento da formação profissional frente ao modelo de organização da atenção à saúde preconizado no SUS.

O presente estudo tem como foco as ações formuladas no âmbito federal direcionadas para a formação dos profissionais de nível superior na área da saúde, envolvendo a articulação entre organizações acadêmicas, de gestão e prestação de serviços. Partindo-se do pressuposto de que as instituições condicionam as preferências e as escolhas dos atores e que as trajetórias das políticas são dependentes de arranjos institucionais e processos decisórios anteriores ${ }^{10}$, propõe-se examinar os marcos e as transformações operadas no percurso temporal da política desde os anos 1980. Busca-se identificar as conexões existentes entre a política desenvolvida a partir de 2003 e as que a antecederam, ressaltando-se os fatores condicionantes e os elementos de continuidade e mudanças.

Os resultados do estudo são apresentados em quatro momentos que demarcam inflexões e conformam modelos de atuação do Executivo Nacional na política de formação profissional em saúde: antecedentes à política de reorientação da formação profissional em saúde - 1980 até o início dos anos 2000; as primeiras experiências desenvolvidas a partir de 2003; e o período mais recente da política, desmembrado em dois momentos - 2005 e 2006, e 2007 a 2010.

\section{Metodologia}

Em termos metodológicos o trabalho ancora-se no referencial de análise de políticas públicas ${ }^{11} \mathrm{e}$, particularmente, na abordagem do institucionalismo histórico ${ }^{10,12,13}$. Segundo essa concepção, as instituições compõem um conjunto de procedimentos, protocolos, normas e convenções oficiais e oficiosas que, além de serem estruturantes e alterarem as estratégias dos atores, condicionam suas preferências ${ }^{13,14}$. Estas não se constituem apenas em arenas políticas, e sim, em atores inseridos no processo político, responsáveis pelo conjunto de estruturas e procedimentos a partir do qual se definem e defendem interesses ${ }^{15}$.

Segundo a posição aqui adotada as instituições são path dependent quando, uma vez iniciada a trajetória de uma política, arranjos institucionais se estabelecem, diminuindo a possibilidade de reversão das escolhas inicialmente fei$\operatorname{tas}^{10}$, o que significa dizer que: "as políticas definidas em um momento no tempo estruturam o processo de tomada de decisão e criam fortes coalizões de suporte que efetivamente protegem os arranjos institucionais estabelecidos, tendo efeitos de feedback no processo político" ${ }^{15}$. A sequência dos acontecimentos também possui relevância no enfoque do institucionalismo histórico; decisões tomadas deixam marcas, criam rotas e dão rumo a procedimentos futuros. 
A partir da vertente histórica do institucionalismo, foram selecionadas algumas categorias de análise que permitiram identificar elementos constitutivos das políticas anteriores e da política em curso e suas inter-relações. São eles: programas e projetos priorizados; objetos preconizados; atores e organizações mobilizadas (seus papéis e relações); estratégias e instrumentos de implementação (incentivos, instâncias e mecanismos de condução, controle e avaliação da política); evidências de implantação.

As estratégias metodológicas envolveram revisão da literatura sobre o tema, que incluiu a busca por estudos de análises de políticas de formação em saúde no Brasil no período de 1980 a 2010. Realizou-se levantamento e análise de documentos relativos ao período mais recente da política nacional (2003 a 2010) compreendendo sua base legal e normativa: portarias e editais de seleção, manuais, relatórios técnicos, apresentações e documentos oficiais e textos de apoios.

O uso da linha do tempo se constitui em um importante recurso metodológico, de modo a ilustrar a trajetória da política em questão. Também permitiu a identificação de inflexões que ajudaram a demarcar momentos específicos do percurso da política e interpretá-los segundo suas principais características.

A pesquisa foi aprovada pelo Comitê de Ética da organização acadêmica envolvida.

\section{Integração docente-assistencial! Redes de projetos e papel das universidades (1980 a 2002)}

As preocupações com a formação de recursos humanos para o setor público de saúde esteve presente no cenário político de concepção do Sistema Único de Saúde, incluindo na Constituição Federal de 1988, a atribuição da saúde em ordenar a formação dos profissionais da área. $\mathrm{O}$ tema compôs as proposições do movimento da Reforma Sanitária, presentes na $8^{\text {a }}$ Conferência Nacional de Saúde e na I Conferência Nacional de Recursos Humanos para a Saúde, em 1986, sinalizando a necessidade de modificação nas graduações e a importância da integração ensino-serviço.

Em 1981, o Programa de Integração Docente Assistencial (IDA), criado no âmbito do Ministério da Educação, desenvolvia projetos de formação profissional por meio da inserção dos alunos em unidades de atenção primária, favorecendo a articulação entre academia e serviços de saúde $^{16}$. O IDA contou com 86 projetos distribu- ídos por 9 redes de programas no Brasil e em outros países da América Latina, ao longo da década ${ }^{17}$. Apesar dos avanços, expressos na conformação da Rede IDA - Brasil, da qual surgiram inúmeras experiências no país, o IDA limitou-se à assistência em saúde, com baixa participação de docentes e segmentação das ações, não resultando em significativas transformações nos currículos $^{18}$.

No início dos anos 90, surge o Projeto UNI Uma Nova Iniciativa, financiado pela Fundação Kellogg, a fim de redimensionar questões relativas ao Programa IDA. As ações orientavam a formação na perspectiva da multiprofissionalidade, do fortalecimento dos componentes curriculares (estágios obrigatórios na comunidade, ênfase em disciplinas que utilizassem a epidemiologia) e da aposta no componente dos serviços representado pelos Sistemas Locais de Saúde (Silos $)^{18}$. O UNI reforçava que os processos de mudança continuassem a ocorrer na comunidade, como forma de superar a dissociação entre proposições acadêmicas e sua aplicabilidade prática $^{19}$. No Brasil, foram implementados 6 projetos UNI, envolvendo 9 profissões de saúde e articulando um amplo conjunto de serviços de saúde, universidades e organizações comunitárias. Os projetos mobilizaram centenas de docentes e beneficiaram milhares de estudantes ${ }^{20}$.

A despeito de projetos como IDA e UNI e da preocupação com a formação no debate sobre a política de saúde no Brasil, a implementação do SUS ocorre na década de 90 e a atuação do Ministério da Saúde na ordenação da formação dos profissionais de saúde foi bastante limitada, sem evidências de uma articulação mais substantiva entre os Ministérios da Saúde e Educação que viabilizasse mudanças curriculares e atendesse às necessidades do sistema de saúde ${ }^{2}$.

Mesmo reconhecendo um contexto desfavorável a políticas mais abrangentes e democratizantes como o SUS, Teixeira e Paim ${ }^{21}$ observam um processo participativo nas universidades neste mesmo período. As ações da Rede Unida, constituída do ideário presente nos projetos IDA e UNI, são exemplos deste processo. A rede contribuiu para a reinserção da temática dos recursos humanos na construção das políticas de saú$\mathrm{de}^{21}$, articulando atores e instituições em torno de mudanças e novas experiências para a formação profissional em saúde ${ }^{19}$.

Nos primeiros anos 2000, foram desenvolvidas pela Comissão Interinstitucional Nacional de Avaliação das Escolas Médicas (CINAEM) iniciativas direcionadas principalmente para trans- 
formação da graduação em medicina, em decorrência dos projetos implantados na década anterior $^{19}$. Em 2002, foi criado o Programa Nacional de Incentivo a Mudanças Curriculares nos Cursos de Medicina (PROMED), desenhado pelos Ministérios da Saúde, da Educação e a OPAS, além da parceria com a Associação Brasileira de Educação Médica (Abem) e a Rede Unida. O programa propunha ações no sentido de adequar a formação médica aos preceitos do SUS, provendo cooperação técnica às reformas curriculares e incentivando a oferta de estágios nos hospitais universitários e nos serviços de atenção básica à saúde $^{22}$. Ao final de 2002, o programa havia contemplado propostas de 19 escolas médicas com financiamento na ordem de oito milhões de reais até o ano de $2003^{22,23}$.

\section{As experiências de reorientação da formação profissional em saúde a partir de 2003}

Em 2003, a criação da Secretaria de Gestão do Trabalho e da Educação na Saúde (SGTES) viabilizou o desenho de políticas para reorientar a formação profissional para o SUS, intensificando, dentre outras ações, o estreitamento das relações entre instituições formadoras e o sistema público de saúde. No período entre 2003 e 2004, é lançado o Projeto Vivências e Estágios na Realidade do Sistema Único de Saúde - o VER-SUS/ Brasil, cujo desenho reconhecia o sistema de saúde como espaço de ensino e aprendizagem. A sua implementação contou com um projeto piloto, no início de 2004, que envolveu 10 cidades da Rede de Municípios colaboradores de Educação Permanente em Saúde e 99 estudantes. Em 2005, duas edições do projeto VER-SUS/Brasil envolveram, respectivamente, cerca de 50 municípios de 19 estados e milhares de estudantes; e $10 \mathrm{mu}$ nicípios de seis estados, com aproximadamente 250 estudantes $^{18}$.

Ainda em 2004, foi lançado o AprenderSUS que teve papel relevante no debate em torno da integralidade da atenção à saúde como eixo de mudança da formação profissional ${ }^{24}$. Uma ampla articulação do Departamento de Gestão da Educação na Saúde (DEGES) da SGTES com a ENSP e a Rede Unida possibilitou a realização do Curso de Especialização de Ativadores de $\mathrm{Mu}-$ danças nas Profissões de Saúde, orientado conceitualmente pelo currículo integrado, o construtivismo e as metodologias educacionais ativas e de educação a distância ${ }^{19}$. Até o ano de 2006, foram formados 597 especialistas ativadores de mudanças em todo o país com recursos financeiros executados na ordem de nove milhões de reais. Atualmente, o Curso de Ativadores de Mudança é oferecido pela Fundação Oswaldo Cruz, por meio do Programa Universidade Aberta do Brasil (UAB) do Ministério da Educação ${ }^{25}$.

No âmbito do AprenderSUS, o projeto denominado EnsinaSUS, contemplou uma série de pesquisas e experiências inovadoras de mudanças na formação e educação permanente em saúde, fornecendo referenciais teóricos para o campo ${ }^{26,27}$. O Fórum Nacional de Educação das Profissões na Área da Saúde (FNEPAS), criado em 2004, organizou-se como importante espaço de debates da formação em saúde ${ }^{19}$, indicando ampliação do conjunto de atores e instituições mobilizados.

A Política Nacional de Educação Permanente em Saúde (PNEPS), de fevereiro de 2004, ocupa posição transversal na articulação de estratégias de mudanças nos processos educacionais em saúde. Naquele ano, as diretrizes da política propuseram a implantação do "Polo de Educação Permanente para o SUS”, reunindo representantes da gestão e da área da educação, com atribuição de detectar problemas e elaborar projetos voltados para a formação e desenvolvimento dos trabalhadores da saúde em um território ${ }^{8}$.

$\mathrm{Na}$ linha do tempo (Figura 1) é possível visualizar a cronologia das principais ações que compõem a evolução da política nacional de reorientação da formação profissional em saúde, desde os seus antecedentes (anos 80/90 e 2002) e as primeiras experiências no âmbito da SGTES até o período mais recente, delimitado pelos anos de 2005 e 2010.

\section{Protagonismo universitário na integração ensino-serviço (2005 a 2006)}

Em 2005, a partir das experiências acumuladas com as iniciativas prévias e maior aproximação entre a saúde e a educação, é instituída a Portaria conjunta no 2.118 , estruturando a parceria entre os Ministérios da Saúde e da Educação "para cooperação técnica na formação e desenvolvimento de recursos humanos na área da saú$\mathrm{de}{ }^{28}$. Inspirado no PROMED, no mesmo ano, é instituído o Programa Nacional de Reorientação da Formação Profissional em Saúde (Pró-Saúde), delineado em um contexto de grandes desafios na implementação das estratégias reformuladoras da formação para o SUS. Inicialmente, o programa (Pró-Saúde I) contemplava os cursos de Enfermagem, Medicina e Odontologia ${ }^{29}$. A inspiração do Pró-Saúde no modelo do PRO- 
Figura 1. Cronologia das principais ações de reorientação da formação profissional em saúde para o SUS. Brasil, décadas de 1980 e 90 e anos 2000.

Fonte: Elaboração dos autores, 2011

MED teve como uma das suas motivações as mudanças ocorridas no DEGES e na SGTES, em virtude da substituição do Ministro da Saúde e de dirigentes do departamento e da secretaria ${ }^{19}$.

A iniciativa tem como foco a aproximação da academia com os serviços públicos de saúde, sustentada na reorientação da formação profissional e na abordagem integral do processo saúdedoença a partir da atenção básica. Os objetivos incluem o estabelecimento de mecanismos de cooperação técnica entre gestores do SUS e as instituições acadêmicas e a ampliação da duração da prática educacional nos serviços do SUS, dentre outros ${ }^{30,31}$.

Com a implementação do Pró-Saúde, esperava-se a substituição do modelo de formação individual, de caráter fortemente especialista e hospitalocêntrico - para um processo formativo que levasse em conta os aspectos socioeconômicos e culturais da população. Preconizava-se a articulação com o sistema público de saúde por meio de ações de promoção da saúde e prevenção de agravos; da difusão da educação profissional como um processo permanente; da busca pelo equilíbrio entre a excelência técnica e os fatores de ordem social; e das mudanças no desenvolvimento das pesquisas em saúde em prol do SUS. Tais argumentos, dentre outros, se constituem nas bases para o desenvolvimento dos projetos institucionais que o programa propõe $\mathrm{e}^{32}$.

A adesão ao Pró-Saúde I era feita por submissão de projeto (um projeto por curso), acompa-
A política nacional de reorientação

da formação profissional em saúde

experiências universitário aprimoramento

VerSUS

PRÓ-SAÚDE I

PRÓ-SAÚDE II

PET-SAÚDE

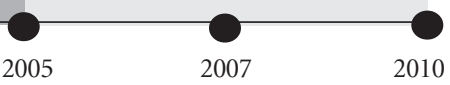

Política Nacional

de Educação Permanente em Saúde nhado de uma carta-compromisso da Secretaria Municipal de Saúde (SMS) e apreciado pelos colegiados dos cursos de graduação. A seleção de propostas ficava a cargo da comissão assessora nacional que avaliava a sua conformidade com as Diretrizes Curriculares Nacionais (DCN) ${ }^{29}$.

Como mecanismos de condução nacional, acompanhamento e avaliação, tem-se a constituição de um conselho consultivo, composto de órgãos e instituições governamentais, do movimento estudantil, conselhos e associações profissionais, e as comissões assessora e executiva, responsáveis pela administração do programa, apoio técnico e avaliação da execução da política, bem como ações de seleção, acompanhamento e avaliação dos projetos. O financiamento ficava a cargo da programação orçamentária do Ministério da Saúde ${ }^{29}$.

Os anos 2005 e 2006 configuraram os passos iniciais da política, evidenciando a maior preocupação em operar os seus dispositivos estruturais e administrativos - composição das comissões e conselho consultivo neste primeiro momento do Pró-Saúde (Pró-Saúde I) - quando se observa a restritividade quanto ao escopo de profissões contempladas pela iniciativa. Destaca-se o protagonismo das Instituições de Ensino Superior (IES) na formulação e implementação das propostas e na estruturação das instâncias de acompanhamento e avaliação do programa. 


\section{Ampliação e aprimoramento da reorientação da formação profissional em saúde (2007 a 2010)}

A partir de 2007, são operadas significativas transformações na trajetória da política nacional, iniciando um novo momento do seu desenho. É neste ano que se cria a Comissão Interministerial de Gestão da Educação na Saúde e que são redefinidas as diretrizes de implantação da Política Nacional de Educação Permanente em Saúde, decorrente da criação dos Colegiados de Gestão Regional (CGR), no interior dos quais se estruturam as Comissões de Integração EnsinoServiço (CIES). As CIES são comissões intersetoriais e interinstitucionais permanentes que apóiam os CGR na elaboração e implantação do Plano de Ação Regional de Educação Permanente em Saúde ${ }^{30}$.

Em 2007, o Pró-Saúde é ampliado para os demais cursos da área de saúde (Pró-Saúde II), os quais, em parceria com as secretarias municipal e estadual de saúde, estruturariam um projeto institucional para adesão ao programa ${ }^{30}$. Prevê-se a constituição das Comissões Estadual e Local de Gestão e Acompanhamento do programa, reunindo representantes da gestão estadual, municipal e dos Conselhos de Saúde, além dos coordenadores dos projetos e dos docentes e discentes envolvidos. Ficam mantidas as estruturas de condução nacional já existentes (comissões assessora e executiva e o conselho consultivo) com ajustes de papéis na comissão executiva, agora responsável pelo edital de seleção de propostas. Permanece também o financiamento a cargo dos recursos orçamentários do Ministério da Saúde, via repasse 'fundo a fundo', além de convênios e demais instrumentos de repasse ${ }^{31}$.

O Pró-Saúde I contemplou 90 cursos das 181 propostas candidatas, sendo 38 de medicina, 27 de enfermagem e 25 de odontologia 9 . Os projetos receberam recursos financeiros num total de nove milhões de reais, repassados para os fundos de saúde de 49 municípios participantes em todo o Brasil ${ }^{33}$. No Pró-Saúde II, 68 projetos foram aprovados, contemplando 354 cursos da área de saúde. Os recursos financeiros compreenderam cerca de cinquenta e três milhões de reais, envolvendo 77 secretarias de saúde ${ }^{34}$. É possível evidenciar a ampliação ocorrida no programa, registrada, pelo maior número de cursos contemplados (quase quatro vezes mais cursos em 2007, em relação a 2005) e pelo aumento no volume de recursos financeiros disponibilizados, bem como a maior participação de secretarias de saúde na construção e implantação dos projetos e ações (Tabela 1).

Também em 2007, surge o Programa de Educação pelo Trabalho para a Saúde (Pet-Saúde) no bojo dos avanços obtidos com o Pró-Saúde, fortalecendo ainda mais a parceria interministerial saúde e educação. O programa tem o foco na qualificação de estudantes de graduação e de pósgraduação, na rede de serviços, por meio de vivências, estágios, iniciação ao trabalho e programas de aperfeiçoamento e especialização. Evidencia ainda a necessidade de incentivos aos profissionais e docentes e destaca a importância das necessidades dos serviços se tornarem objeto de pesquisa e fonte de produção do conhecimento nas instituições acadêmicas ${ }^{36,37}$.

A proposta do Pet-Saúde é favorecer a indissociabilidade entre ensino, pesquisa e extensão, a constituição de grupos de aprendizagem tutorial, a interdisciplinaridade e a integração ensinoserviço, incluindo um plano de pesquisa em atenção básica ${ }^{38}$. O grupo PET é formado por um tutor acadêmico que coordena atividades de 30 alunos, sendo 12 deles bolsistas de iniciação ao trabalho, orientados por preceptores nos serviços de saúde ${ }^{38,39}$.

Os projetos para adesão ao programa são assinados conjuntamente pelo gestor municipal e pela instituição de ensino superior (IES), res-

Tabela 1. Número de cursos e projetos contemplados, IES e Secretarias de Saúde envolvidas e total de recursos financeiros empregados nas duas edições do Programa Nacional de Reorientação da Formação Profissional em Saúde - PRÓ-SAÚDE. Brasil, 2010.

\begin{tabular}{lccccc}
\hline & $\begin{array}{c}\text { Cursos } \\
\text { contemplados }\end{array}$ & $\begin{array}{c}\text { Projetos } \\
\text { contemplados }\end{array}$ & $\begin{array}{c}\text { Secretarias } \\
\text { envolvidas }\end{array}$ & $\begin{array}{c}\text { Recursos } \\
\text { de saúde } \\
\text { envolvidas }\end{array}$ & $\begin{array}{c}\text { financeiros (em } \\
\text { milhões de reais) }\end{array}$ \\
\hline PRÓ-SAÚDE I (2005) & 90 & 90 & 62 & 49 & 9 \\
PRÓ-SAÚDE II (2007) & 354 & 68 & 68 & 77 & $53^{*}$ \\
\hline
\end{tabular}

${ }^{*}$ Valor aproximado

Fontes: Brasil $1^{33,34,35}$ 
ponsáveis também pela definição dos critérios de seleção dos preceptores. As propostas são apreciadas nas Comissões de Integração EnsinoServiço e pactuadas na Comissão Intergestores Bipartite (CIB) e no Conselho Municipal de Saúde $(\mathrm{CMS})^{38}$. Em 2010, o programa passa por mudanças possibilitando o desenvolvimento de grupos PET temáticos em Saúde da Família, Vigilância em Saúde e Saúde Mental ${ }^{37}$.

A condução nacional do Pet-Saúde fica a cargo do DEGES/SGTES, que tem a atribuição de apreciar as propostas, sugerir mecanismos de funcionamento, aprimoramento e avaliação dos grupos PET. As instituições de ensino superior têm a atribuição de selecionar os estudantes e os tutores acadêmicos e as secretarias de saúde indicam os nomes dos preceptores. A Comissão de Avaliação do Pet-Saúde se manifesta em relação ao desempenho dos grupos tutoriais e da necessidade de expansão ou extinção dos mesmos ${ }^{38}$.

Os incentivos à implementação envolvem a oferta de bolsas aos alunos participantes, aos docentes das IES e aos preceptores nos serviços de saúde ${ }^{37}$. A concessão das bolsas está condicionada à constituição e manutenção, sob atribuição da IES, dos Núcleos de Excelência Clínica Aplicada à Atenção Básica ${ }^{36}$. A entrada/desligamento de bolsistas, suspensão e concessão de bolsas, bem como o acompanhamento mensal dos pagamentos é realizado pelo Sistema de Informações Gerenciais do programa - SIG-Pet-Saúde ${ }^{40}$.

No Pet-Saúde, foram aprovados 84 projetos de 68 instituições de ensino superior em parceria com 71 secretarias de saúde, tendo sido constituídos 306 grupos PET, em 2009, quando onze mil estudantes participaram do programa, com cerca de cinco mil bolsas concedidas ${ }^{9,41,42}$. Em 2010, aprovou-se 111 projetos, envolvendo 84 IES e 96 secretarias de saúde, e 461 grupos tutoriais (neste ano, já sob a denominação de 'Saúde da Família'). Em maio do mesmo ano foram mais 70 projetos e 122 grupos PET temáticos de Vigilância em Saúde (VS), envolvendo 60 IES e 59 secretarias de saúde.

Quanto às bolsas concedidas, o PET-Saúde/ Saúde da Família contou com 8.700 bolsas/mês e cerca de dezessete mil estudantes, preceptores e tutores. O PET-Saúde/VS concedeu cerca de 1.300 bolsas/mês no ano de $2010^{43}$. Nesse sentido, observa-se, entre 2009 e 2010, significativa ampliação do programa, com aumento no número de projetos aprovados, na quantidade de atores e instituições mobilizados e nos incentivos concedidos por meio das bolsas (Tabela 2).

Dessa forma, é possível observar a ampliação e o aprimoramento das bases estruturantes da política. O marco legal e normativo analisado indica maior valorização das instâncias decisórias do SUS, reduzindo o protagonismo acadêmico presente no momento anterior. Destaca-se a mobilização dos atores das gestões estadual e municipal na implementação local da política, com ativa participação na construção conjunta das propostas, no estabelecimento de parcerias e na composição de comissões estadual e local de gestão dos projetos. Outro aspecto é a inclusão dos programas nas discussões dos distintos espaços de condução da política de saúde estadual e local (SES, SMS, CES, CMS etc.), bem como de representantes destas instâncias na implementação das propostas. São também relevantes os incrementos nos recursos financeiros para a readequação dos serviços de saúde e o aprimoramento dos instrumentos e aspectos conceituais da política, na busca pelo fortalecimento da integração ensino-serviço-comunidade.

O Pet-Saúde, especificamente, passou por uma sucessiva incorporação de novas diretrizes e ajustes dos instrumentos de implementação. Em sintonia ao processo de valorização de novos atores e instâncias do SUS, iniciado no Pró-Saúde, o programa teve maior articulação com a Secretaria de Atenção à Saúde do Ministério da Saúde (SAS/MS); valorizou o profissional dos

Tabela 2. Número de projetos contemplados, grupos PET constituídos, IES e Secretarias de Saúde envolvidas, bolsas concedidas e participantes do Programa de Educação pelo Trabalho para a Saúde - PET-SAÚDE. Brasil, 2010.

\begin{tabular}{|c|c|c|}
\hline & 2009 & $2010^{*}$ \\
\hline Projetos contemplados & 84 & 181 \\
\hline Grupos constituídos & 306 & 583 \\
\hline IES participantes & 68 & 144 \\
\hline Secretarias de Saúde envolvidas & 71 & $164^{* *}$ \\
\hline Bolsas concedidas & 5.814 & 10.101 \\
\hline \multicolumn{3}{|l|}{ Participantes } \\
\hline Tutores & 306 & 583 \\
\hline Preceptores & 1.836 & 3.010 \\
\hline Estudantes bolsistas & 3.672 & 6.508 \\
\hline Estudantes não bolsistas & 5.508 & $8.298^{* * *}$ \\
\hline Total & 11.322 & 18.399 \\
\hline
\end{tabular}

* Para o biênio 2010/2011, números e valores agrupados para os grupos PET-SAÚDE/Saúde da Família e PET-SAÚDE/ Vigilância em Saúde. ${ }^{* *}$ Valor global. Pode haver duplicidade. ${ }^{* * *}$ Número de não bolsistas correspondente aos grupos Pet-Saúde/Saúde da Família. Para o Pet-Saúde/ Vigilância em Saúde não há registros de estudantes não bolsistas.

Fontes: Brasil ${ }^{35,41,42}$. 
serviços de saúde e os alunos de graduação; e inseriu-se nas Comissões de Integração EnsinoServiço (CIES) dos CGR ${ }^{37,38}$. A partir de 2010, a criação dos PET temáticos sinaliza para novos rumos da política, indicando o início de um terceiro momento de sua institucionalização, pautado pelo avanço na diversificação de atores e instâncias nas variadas frentes de atuação do SUS, para além da atenção básica à saúde.

O Quadro 1 resume os principais aspectos dos momentos apresentados, destacando, para cada um deles, os programas e projetos prioritários, objetos, atores e organizações, estratégias e instrumentos, bem como evidências de implantação.

\section{Considerações finais}

A análise dos distintos momentos destacados no percurso temporal da política nacional de reorientação da formação profissional em saúde evi- dencia conexões que fomentam o desenvolvimento dos programas e projetos implementados numa perspectiva de continuidade dos eixos norteadores da reorientação da formação para o SUS. O fortalecimento da integração ensino-serviço-comunidade e a centralidade na atenção básica à saúde são alguns dos componentes presentes nos objetos preconizados pelas iniciativas, bem como norteiam as principais estratégias e instrumentos delineados.

Tais conexões sinalizam para os arranjos institucionais que se estabelecem na sequência dos acontecimentos, ocasionando o aparecimento de feedbacks nos novos projetos e programas desenvolvidos no âmbito da política. Neste cenário, destacam-se a construção dos projetos UNI a partir dos limites e possibilidades do Programa IDA e, mais recentemente, da associação entre o Pró-Saúde e os elementos que compuseram o PROMED. As evidências de implantação das ações expressam os sucessivos incrementos in-

Quadro 1. Principais momentos da trajetória da política nacional de reorientação da formação profissional em saúde, dos anos 1980 a 2010: programas e projetos prioritários, objetos, atores e organizações, estratégias e instrumentos e principais evidências de implementação

\begin{tabular}{|c|c|c|c|}
\hline Momento & \multicolumn{3}{|c|}{ Antecedentes } \\
\hline Período/ano & \multicolumn{2}{|c|}{ Anos 80 e 90} & 2002 \\
\hline $\begin{array}{l}\text { Programas e } \\
\text { projetos } \\
\text { prioritários }\end{array}$ & IDA & UNI & PROMED \\
\hline $\begin{array}{l}\text { Objetos da } \\
\text { política }\end{array}$ & $\begin{array}{l}\text { Integração docente } \\
\text { assistencial }\end{array}$ & $\begin{array}{l}\text { Multiprofissionalidade } \\
\text { Currículo de graduação } \\
\text { Ensino-serviço- } \\
\text { comunidade }\end{array}$ & $\begin{array}{l}\text { Formação médica para o SUS } \\
\text { Reforma curricular }\end{array}$ \\
\hline $\begin{array}{c}\text { Atores e } \\
\text { organizações } \\
\text { mobilizados }\end{array}$ & $\begin{array}{l}\text { Universidades e serviços } \\
\text { de saúde }\end{array}$ & $\begin{array}{l}\text { Docentes e estudantes } \\
\text { universitários } \\
\text { Universidades, Serviços de } \\
\text { Saúde e comunidade }\end{array}$ & $\begin{array}{l}\text { Estudantes e docentes de } \\
\text { medicina } \\
\text { Universidades }\end{array}$ \\
\hline $\begin{array}{l}\text { Estratégias e } \\
\text { instrumentos }\end{array}$ & $\begin{array}{l}\text { Projetos para inserção } \\
\text { na prática do cuidado } \\
\text { Rede IDA-Brasil }\end{array}$ & $\begin{array}{l}\text { Estágios na comunidade } \\
\text { Ênfase em disciplinas como } \\
\text { a epidemiologia } \\
\text { Rede UNIDA }\end{array}$ & $\begin{array}{l}\text { Cooperação técnica às } \\
\text { reformas curriculares } \\
\text { Incentivos a estágios }\end{array}$ \\
\hline $\begin{array}{l}\text { Evidências de } \\
\text { implementação }\end{array}$ & $\begin{array}{l}86 \text { projetos } \\
9 \text { redes de programas na } \\
\text { América Latina } \\
\text { Limites: baixa } \\
\text { participação docente, } \\
\text { foco na assistência e } \\
\text { segmentação }\end{array}$ & $\begin{array}{l}6 \text { projetos UNI } \\
\text { desenvolvidos envolvendo } 9 \\
\text { graduações de saúde }\end{array}$ & $\begin{array}{l}\text { Implantado em } 19 \text { escolas } \\
\text { médicas brasileiras } \\
8 \text { milhões de reais empregados } \\
\text { Implantação apenas } \\
\text { em cursos de medicina }\end{array}$ \\
\hline
\end{tabular}


Quadro 1. continuação

\begin{tabular}{|c|c|c|}
\hline Momento & \multicolumn{2}{|c|}{ Política nacional de reorientação da formação profissional em saúde } \\
\hline & \multicolumn{2}{|c|}{ Primeiras experiências } \\
\hline Período/ano & \multicolumn{2}{|r|}{2003 e 2004} \\
\hline $\begin{array}{l}\text { Programas e } \\
\text { projetos } \\
\text { prioritários }\end{array}$ & VER-SUS & AprenderSUS \\
\hline $\begin{array}{l}\text { Objetos da } \\
\text { política }\end{array}$ & Ensino-aprendizagem no SUS & $\begin{array}{l}\text { Integralidade da atenção } \\
\text { Currículo integrado } \\
\text { Construtivismo } \\
\text { Metodologias ativas } \\
\text { EAD }\end{array}$ \\
\hline $\begin{array}{l}\text { Atores e } \\
\text { organizações } \\
\text { mobilizados }\end{array}$ & $\begin{array}{l}\text { Estudantes e docentes } \\
\text { Universidades e serviços de saúde }\end{array}$ & $\begin{array}{l}\text { Estudantes, docentes e profissionais de saúde } \\
\text { Universidades e serviços de saúde }\end{array}$ \\
\hline $\begin{array}{l}\text { Estratégias e } \\
\text { instrumentos }\end{array}$ & $\begin{array}{l}\text { Vivências na realidade } \\
\text { locorregional }\end{array}$ & $\begin{array}{l}\text { Curso Ativadores de Mudanças } \\
\text { Pesquisas: EnsinaSUS }\end{array}$ \\
\hline $\begin{array}{l}\text { Evidências de } \\
\text { implementação }\end{array}$ & 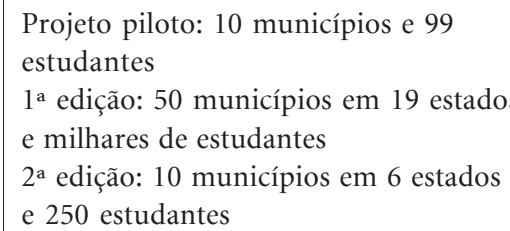 & $\begin{array}{l}597 \text { concluintes na 1a edição do Curso de } \\
\text { Especialização em Ativadores de Mudanças } \\
\text { Recursos financeiros na ordem de } 9 \text { milhões } \\
\text { de reais } \\
\text { Curso reeditado pelo Programa UAB (MEC) }\end{array}$ \\
\hline \multirow[t]{2}{*}{ Momento } & \multicolumn{2}{|c|}{ Política nacional de reorientação da formação profissional em saúde } \\
\hline & Passos iniciais de institucionalização & Ampliação e aprimoramento \\
\hline Período/ano & 2005 e 2006 & 2007 a2010 \\
\hline $\begin{array}{l}\text { Programas e } \\
\text { projetos } \\
\text { prioritários }\end{array}$ & PRÓ-SAÚDE I & $\begin{array}{l}\text { PRÓ-SAÚDE II } \\
\text { PET-SAÚDE }\end{array}$ \\
\hline $\begin{array}{l}\text { Objetos da } \\
\text { política }\end{array}$ & $\begin{array}{l}\text { Integração ensino-serviço } \\
\text { Atenção integral à saúde } \\
\text { Atenção Básica }\end{array}$ & $\begin{array}{l}\text { Integração ensino-serviço-comunidade } \\
\text { Aprendizagem tutorial } \\
\text { Capacitação docente } \\
\text { Produção acadêmica - necessidades dos serviços }\end{array}$ \\
\hline $\begin{array}{l}\text { Atores e } \\
\text { organizações } \\
\text { mobilizados }\end{array}$ & $\begin{array}{l}\text { Estudantes e docentes } \\
\text { Universidades, Secretarias e } \\
\text { Serviços de Saúde }\end{array}$ & $\begin{array}{l}\text { Estudantes, docentes, profissionais de saúde } \\
\text { Universidades, Secretarias e Serviços de Saúde } \\
\text { Instâncias colegiadas do SUS: CIB, CGR e CIES }\end{array}$ \\
\hline $\begin{array}{l}\text { Estratégias e } \\
\text { instrumentos }\end{array}$ & $\begin{array}{l}\text { Adesão por meio de projetos } \\
\text { Comissão assessora e executiva } \\
\text { Conselho consultivo } \\
\text { Incentivos financeiros } \\
\text { Acompanhamento e avaliação } \\
\text { por instâncias nacionais }\end{array}$ & $\begin{array}{l}\text { Adesão por meio de projetos } \\
\text { Instâncias nacionais, regionais e locais de } \\
\text { acompanhamento e avaliação } \\
\text { Pagamentos de bolsas SIG-PET-SAÚDE } \\
\text { Núcleos de Excelência Clínica na Atenção } \\
\text { Básica }\end{array}$ \\
\hline $\begin{array}{l}\text { Evidências de } \\
\text { implementação }\end{array}$ & $\begin{array}{l}\text { Restrito aos cursos de Medicina, } \\
\text { Enfermagem e Odontologia } \\
\text { Protagonismo universitário } \\
\text { Foco nos aspectos estruturais } \\
\text { e administrativos da implantação }\end{array}$ & $\begin{array}{l}\text { Ampliação para as demais profissões de saúde } \\
\text { Valorização das instâncias decisórias do SUS e } \\
\text { da função de tutoria e preceptoria } \\
\text { Incremento nos incentivos financeiros } \\
\text { Valorização das instâncias de condução } \\
\text { locorregional }\end{array}$ \\
\hline
\end{tabular}

Fonte: Elaboração dos autores, 2011. 
corporados às iniciativas, à luz de experiências anteriores, sugerindo a ampliação da política, com o aumento e a diversificação dos atores e organizações envolvidos e o maior quantitativo de projetos implementados. Os avanços quanto ao desenho das ações também se apresentam associados às experiências acumuladas ao longo do tempo, representando o amadurecimento de suas bases estruturantes.

A trajetória da política estudada é marcada pela crescente aproximação entre os Ministérios da Saúde e da Educação, observada a sua maior institucionalidade a partir de 2003. Tal contexto propicia efetivar o ordenamento da formação dos recursos humanos para o SUS, por meio da ope- racionalização conjunta de políticas para o setor. No conjunto das mudanças operadas, com destaque para o ano de 2007, destaca-se a maior participação das estruturas de gestão do sistema de saúde (secretarias estadual e municipal) e dos profissionais dos serviços no processo político e decisório em que se concebem os projetos. Além disso, as ações recentes de reorientação da formação profissional vêm sendo delineadas em instâncias loco - regionais do Sistema Único de Saúde, como os Colegiados de Gestão Regional, relacionando-se mais efetivamente ao processo de regionalização e descentralização da política nacional de saúde.

\section{Colaboradores}

Os autores HSA Dias, LD Lima e M Teixeira foram responsáveis pela concepção, desenvolvimento, análise das informações, redação e revisão do artigo.

\section{Agradecimentos}

Agradecemos ao Conselho Nacional de Desenvolvimento Científico e Tecnológico - CNPq e ao Edital do Proex-Capes-ENSP 2010, pelo apoio financeiro à realização do estudo. LD Lima é Bolsista de Produtividade em Pesquisa do CNPq. 


\section{Referências}

1. Machado MH. Trabalhadores da saúde e sua trajetória na reforma sanitária. In: Lima NT, Gerschman S, Edler FC, Suárez JM, organizadores. Saúde e Democracia: História e Perspectivas do SUS. Rio de Janeiro: Editora Fiocruz; 2005.

2. Machado CV. Direito universal, política nacional: o papel do Ministério da Saúde na política de saúde brasileira de 1990 a 2002. Rio de Janeiro: Editora Museu da República; 2007.

3. Araújo ME, Zilbovicius C. A formação acadêmica para o trabalho no Sistema Único de Saúde (SUS). In: Moysés ST, Kriger L, Moysés SJ, coordenadores. Saúde bucal das famílias - trabalhando com evidências. São Paulo: Artes Médicas; 2008.

4. Haddad AE, Morita MC, Pierantoni CR, Brenelli SL, Passarella T, Campos FE. Formação de profissionais de saúde no Brasil: uma análise no período de 1991 a 2008. Rev Saúde Pública 2010; 44(3):383-393.

5. Campos FE, Pierantoni CR, Machado MH. Introdução. Cadernos RH Saúde 2006; 3(1):8-11.

6. Campos FE, Aguiar RAT, Belisário AS. A formação superior dos profissionais de saúde. In: Giovanella L, Escorel S, Lobato LVC, Noronha JC, Carvalho AI, organizadores. Políticas e sistema de saúde no Brasil. Rio de Janeiro: Editora Fiocruz; 2008.

7. Pierantoni CR, Viana ALD. Apresentação. In: Pierantoni CR, Viana ALD, organizadores. Educação e Saúde. São Paulo: Editora Hucitec; 2010.

8. Brasil. Ministério da Saúde (MS). Portaria GM/MS no 198, de 13 de fevereiro de 2004. Institui a Política Nacional de Educação Permanente em Saúde como estratégia do Sistema Único de Saúde para a formacão e o desenvolvimento de trabalhadores para o setor dá outras providências. Diário Oficial da União 2004; 14 fev.

9. Brasil. Ministério da Saúde (MS). Secretaria de Gestão do Trabalho e da Educação na Saúde. A educação e o trabalho na saúde: a política e suas ações. Brasília: MS; 2009.

10. Pierson P. Politics in Time: History, Institutions and Social Analysis. Princeton University Press; 2004.

11. Ham C, Hill M. The policy process in the modern capitalist State. Hertfordshire: Haverster Wheatsheaf; 1993.

12. Thelen K, Steinmo S. Historical institutionalism in comparative politics. In: Thelen K, Steinmo S, Longstreth F, organizadores. Structuring Politics: historical institutionalism en comparative analysis. Cambridge: Cambridge University Press; 1992. p.1-32.

13. Hall PA, Taylor RCR. As três versões do neo-institucionalismo. Lua Nova 2003; 58:193-223.

14. Marques E. Notas críticas à literatura sobre Estado, políticas estatais e atores políticos. Revista Brasileira de Informação Bibliográfica em Ciências Sociais (BIB)1997; 43:67-102.

15. Menicucci TMG. Os agumentos analíticos: a perspectiva histórica e institucional. In: Menicucci TMG, organizador. Público e privado na política de assistência à saúde no Brasil. Atores, processos e trajetória. Rio de Janeiro: Editora Fiocruz; 2007.
16. Costa HOG, Kalil MEX, Sader NMB. Red UNIDA: Un nuevo actor social em El campo de lãs políticas públicas. In: Almeida M, Feuerwerker L, Llanos C $\mathrm{M}$, editores. La educación de los profesionales de la salud em latinoamerica: teoría y práctica de un movimiento de cambio. São Paulo, Buenos Aires, Londrina: Hucitec, Lugar Editorial, Ed. UEL; 1999.

17. Chaves M, Kisil M. Origens, concepção e desenvolvimento. In: Almeida MJ, Feuerwerker L, Llanos $\mathrm{M}$, organizadores. A educação dos profissionais de saúde na América Latina: teoria e prática de um movimento de mudança. Tomo 1: um olhar analítico. São Paulo: Hucitec; 1999. p. 1-16

18. Torres OM. Os estágios de vivência no Sistema Único de Saúde: das experiências regionais à (trans)formação político-pedagógica do Ver-SUS/Brasil [dissertação]. Salvador: Universidade Federal da Bahia; Salvador.

19. Gonzalez AD, Almeida MJ. Movimentos de mudança na formação em saúde: da medicina comunitária às diretrizes curriculares. Physis 2010; 20(2):551-570.

20. Almeida M, Feuerwerker L, Llanos CM, editores. La educación de los profesionales de la salud em latinoamerica: teoría y práctica de un movimiento de cambio.V. 2. São Paulo, Buenos Aires, Londrina: Hucitec, Lugar Editorial, Ed. UEL; 1999.

21. Teixeira CF, Paim JS. Políticas de formação de recursos humanos em saúde: conjuntura atual e perspectivas. Divulgação em saúde para debate 1996; (12):19-23.

22. Oliveira NA, Meirelles RMS, Cury GC, Alves LA. Mudanças curriculares no ensino médico brasileiro: um debate crucial no contexto do promed. Rev bras educ med 2008; 32(3):333-346.

23. Goulart LMHF, Alves CRL, Belisário SA, Abreu DMX, Lemos JMC, Massote AW, França MB, Mendes KF, Silva TAF. Abordagem pedagógica e diversificação dos cenários de ensino médico: projetos selecionados pelo PROMED. Rev bras educ med 2009; 33(4):605-614.

24. Brasil. Ministério da Saúde (MS). Aprender SUS: O SUS e os cursos de graduação da área de saúde. Brasília: MS; 2004.

25. Ministério da Saúde (MS).Escola Naciona de Saúde Pública (ENSP). Fundação Oswaldo Cruz (Fiocruz)- Rede Unida. Curso de Especialização em Ativação de Processos de Mudanças na Formação Superior de Profissionais de Saúde. [relatório final]. Rio de Janeiro: EAD, ENSP, Fiocruz-Rede UNIDA, SGTES, Ministério da Saúde; 2006.

26. Ceccim RB, Carvalho YM. Ensino da saúde como projeto da integralidade: a educação dos profissionais de saúde no SUS. In: Pinheiro R, Ceccim RB, Mattos RA, organizadores. Ensinar Saúde: a integralidade e o SUS nos cursos de graduação na área da saúde. 2a Edição. Rio de Janeiro: IMS, UERJ, CEPESQ, Abrasco; 2006. 
27. Koifmann L, Henriques RLM. A experiência da pesquisa EnsinaSUS. Trabalho, Educação e Saúde 2007; 5(1):161-172.

28. Brasil. Ministério da Saúde (MS). Ministério da Educação. Portaria no ${ }^{\circ}$. 2.118, de 3 de novembro de 2005. Institui parceria entre o Ministério da Educação e o Ministério da Saúde para cooperação técnica na formação e desenvolvimento de recursos humanos na área da saúde. Diário Oficial da União 2005; 4 nov.

29. Brasil. Ministério da Saúde (MS). Portaria interministerial n. 2.101, de 3 de novembro de 2005. Institui o Programa Nacional de Reorientação da Formação Profissional em Saúde - Pró-Saúde - para os cursos de graduação em Medicina, Enfermagem e Odontologia. Diário Oficial da União 2005; 4 nov.

30. Brasil. Ministério da Saúde (MS). Portaria interministerial no 3.019, de 26 de novembro de 2007. Dispõe sobre o Programa Nacional de Reorientação da Formação Profissional em Saúde - Pró-Saúde para os cursos de graduação da área da saúde. Diário Oficial da União 2008; 23 out.

31. Brasil. Ministério da Saúde (MS). Ministério da Educação (ME). Programa Nacional de Reorientação da Formação Profissional em Saúde - Pró-Saúde: Objetivos, Implementação e Desenvolvimento Potencial. Brasília: MS, ME; 2007.

32. Brasil. Ministério da Saúde (MS). Portaria GM/MS no 1.996 de 20 de agosto de 2007. Dispõe sobre as diretrizes para a implementação da Política Nacional de Educação Permanente em Saúde e dá outras providências. Diário Oficial da União 2007; 22 ago.

33. Brasil. Ministério da Saúde (MS). Portaria conjunta $n^{\circ} 48$ de 30 de junho de 2006. Diário Oficial da União 2006; 12 set.

34. Brasil. Ministério da Saúde (MS). Portaria no 1.282, de 25 de junho de 2008. Define que os valores publicados para a implementação do Programa Nacional de Reorientação da Formação Profissional em Saúde - Pró-Saúde sejam repassados durante os exercícios de 2008, 2009 e 2010 aos respectivos Fundos Estaduais e Municipais de Saúde. Diário Oficial da União 2008; 26 jun.

35. Brasil. Ministério da Saúde (MS). SGTES/MS. [página na Internet]. [acessado 2013 abr 27]. Disponível em: www.portal.saude.gov.br

36. Brasil. Ministério da Saúde (MS). Portaria interministerial $n^{\circ}$. 1.802, de 26 de agosto de 2008. Institui o Programa de Educação pelo Trabalho para a Saúde - PET - Saúde. Diário Oficial da União 2008; 27 ago.

37. Brasil. Ministério da Saúde (MS). Portaria interministerial no 421, de 3 de março de 2010. Institui o Programa de Educação pelo Trabalho para a Saúde (PET-Saúde) e dá outras providências. Diário Oficial da União 2010; 5 mar.
38. Brasil. Ministério da Saúde (MS). Edital no 18 Seleção para o Programa de Educação pelo Trabalho para a Saúde - Pet-Saúde. Brasília: MS; 2009.

39. Brasil. Ministério da Saúde (MS). Portaria Interministerial $n^{\circ} 422$, de 3 de março de 2010. Estabelece orientações e diretrizes técnico-administrativas para a execução do Programa de Educação pelo Trabalho para a Saúde - PET Saúde, instituído no âmbito do Ministério da Saúde e da Educação. Diário Oficial da União 2010; 4 mar.

40. Brasil. Secretaria de Gestão do Trabalho e da Educação na Saúde. Portaria no 4, de 29 de março de 2010. Estabelece orientações e diretrizes para a concessão de bolsas de iniciação ao trabalho, tutoria acadêmica e preceptoria para a execução do Programa de Educação pelo Trabalho para a Saúde PET-Saúde, instituído no âmbito do Ministério da Saúde e do Ministério da Educação. Diário Oficial da União 2010; 30 mar.

41. Brasil. Ministério da Saúde (MS). Ministério da Educação (ME). Portaria conjunta no 3 , de 30 de janeiro de 2009. Homologa o resultado do processo de seleção dos projetos que se candidataram ao Programa de Educação pelo Trabalho para a Saúde - Pet-Saúde. Diário Oficial da União 2009; 31 jan.

42. Brasil. Ministério da Saúde (MS). Ministério da Educação. Portaria conjunta no 4 , de 6 de fevereiro de 2009. Altera a homologação do resultado do processo de seleção dos projetos que se candidataram ao Programa de Educação pelo Trabalho para a Saúde - Pet-Saúde. Diário Oficial da União 2009; 10 fev.

43. Brasil. Secretaria de Gestão do Trabalho e Educação na Saúde. Coordenação PET-Saúde. Projetos. [documento da Internet].[acessado 2013 abr 27]. Disponível em: http://portal.saude.gov.br/portal/saúde/ profissional/ visualizar_texto.cfm?idtxt $=32571$

Artigo apresentado em 20/09/2012

Aprovado em 31/10/2012

Versão final apresentada em 26/11/2012 